\title{
Insight into Photon Recycling in Perovskite Semiconductors from the Concept of Photon Diffusion
}

\author{
Mehdi Ansari-Rad ${ }^{1, *}$ and Juan Bisquert ${ }^{2}$ \\ ${ }^{1}$ Faculty of Physics, Shahrood University of Technology, Shahrood 3619995161, Iran \\ ${ }^{2}$ Institute of Advanced Materials (INAM), Universitat Jaume I, 12006 Castelló, Spain
}

(Received 25 December 2017; revised manuscript received 29 July 2018; published 27 September 2018)

\begin{abstract}
Photon recycling has been proven to be an important process in metal halide perovskite thin films. We propose a model of photon recycling based on the photon diffusion concept that can interpret experimental observations on the photoluminescence and transient spectroscopy in perovskite layers. Our model shows how the photon recycling can enhance the apparent photocarrier lifetime and thereby slow down the photoluminescence decay dynamics. We also discuss the interplay between photon recycling and the photocarriers diffusive transport and demonstrate that at high carrier concentrations, photon recycling dominates the diffusion, conveying the carriers inside the absorbing layer over long distances. We also provide a quantitative determination of a diffusion length that is considerably expanded by the photon recycling process. The effect of the photon recycling process on the Auger recombination rate is also addressed here. The results provide insights for both interpreting the experimental observations and also designing experimental schemes.
\end{abstract}

DOI: 10.1103/PhysRevApplied.10.034062

\section{INTRODUCTION}

Recently, metal halide perovskites have attracted considerable attention for optoelectronic applications owing to their excellent light absorption and electronic properties [1]. Efficient radiative recombination in metal halide perovskites is one the most important properties of these materials, introducing them as good candidates for both photoluminescent and photovoltaic devices. Generally, the photons created by the radiative recombination of the charge carriers may be reabsorbed again before being able to escape from the system and subsequently reemitted. The first evidence on the photon reabsorption in the halide perovskites was reported in the work by Yamada et al. in which the photoluminescence (PL) dynamics in single crystals halide perovskite was explained by the photon reabsorption effect [2]. This process, usually called photon recycling (PR), has been demonstrated to have a unique impact on the charge and energy transport length scales in perovskite layers, and also on the open circuit voltage of the perovskite solar cells [3].

Recently, transient spectroscopy and time-resolved PL measurement have been used to investigate the PR efficiency in perovskite thin films [4-9]. It has been observed in these studies that by changing the light out-coupling feature of the film (via changing the thickness or optical design of the system, for example), one can manipulate the externally measured (apparent) radiative recombination

${ }^{*}$ mehdi.ansari.rad@gmail.com coefficient; an observation that is directly attributed to the PR in the system. A similar result was already reported for GaAs thin films in which thicker devices were observed to show considerably longer PL lifetimes in comparison with the radiative lifetime theoretically expected [10]. However, it should be noted that the PR efficiency for solar cell applications (where the device is irradiated by 1 Sun illumination) has been evaluated to be as low as $<0.5 \%$ [11], although the benefit in the open circuit voltage of the cell from the PR effect has been estimated to be as high as 50-80 mV [12].

The evidence mentioned above suggests that the PR effect cannot be neglected in the advanced simulation of perovskite optoelectronic devices, especially at high carrier densities where the radiative bimolecular recombination becomes the dominant recombination channel. Detailed modeling of the PR has been reported in recent works in which ray optics in combination with the detailed balance considerations are used to calculate the probability of the photon absorption, emission, and losses in the optical system $[6,8,13]$. However, a physically intuitive model in which various absorption and loss mechanisms of the photons and the charge carriers can be easily incorporated into the model is not available for research groups. Here, we present a different approach in which the population of the photons in the system is resolved by a continuity equation coupled to the continuity equation of the charge carriers. Results of the model are in agreement with the experimental trends reported for halide perovskites thin films. 


\section{MODEL AND METHOD}

We consider an absorbing layer of thickness $d$, absorption coefficient $\alpha(E)$, and refractive index $n_{r}$, that is illuminated from the left (the front side) by a monochromatic (continuous or transient) flux $I_{0}$ of photons of energy $E$. For simplicity, the energy of the photons is considered to be near the absorption edge of the layer. However, the model can straightforwardly be extended to an arbitrary (monochromatic or spectral) illumination source. Taking into account the probability of the PR in the system, the continuity equation for the electrons created by the incoming photons inside the layer can be written as (there is also a similar equation for the holes)

$$
\frac{\partial n}{\partial t}=-\frac{\partial j_{n}}{\partial x}+I_{0} \alpha e^{-\alpha x}-B_{\mathrm{rad}}^{\prime} n p-\frac{n}{\tau_{\mathrm{SRH}}}-k_{\mathrm{Agr}} n^{2} p
$$

where $n=n(x, t)$ and $p=p(x, t)$ are the densities of the electrons and holes in the layer, respectively, $j_{n}=$ $-D_{n} \partial n / \partial x$ is the electron mass current with $D_{n}$ being the diffusion coefficient, $B_{\text {rad }}^{\prime}$ is the apparent radiative recombination coefficient, and $\tau_{\mathrm{SRH}}$ is the Shockley-Read-Hall (nonradiative) recombination lifetime. Auger recombination $\left(\propto n^{3}\right)$ is also included in the model via the rate constant $k_{\mathrm{Agr}}$, although it is important only at high charge densities $n \gtrsim 10^{18} \mathrm{~cm}^{-3}$ [14].

Due to the possibility of photon reabsorption after the radiative recombination events, the term $I_{0} \alpha e^{-\alpha x}$ is not a correct generation term in the equation. To account for this, one must use an apparent radiative coefficient, $B_{\text {rad }}^{\prime}$, in the modeling. $B_{\text {rad }}^{\prime}$ is different from the intrinsic radiative recombination coefficient $B_{\text {rad }}$, and is a function of the optical and geometrical properties of the layer. On the other hand, as a result of the detailed balance principle, $B_{\text {rad }}$ is linked to the absorption coefficient of the layer as [15]

$$
B_{\mathrm{rad}}(E) \approx \frac{4 b_{\pi}}{n_{i}^{2}} \alpha(E) E^{2} e^{-\left(E / k_{B} T\right)} d E
$$

where $b_{\pi}=2 \pi n_{r}^{2} / h^{3} c^{2}$ with $h$ being the Planck constant and $c$ the speed of light in the vacuum. $n_{i}^{2}=$ $N_{c} N_{v} \exp \left(-E_{g} / k_{B} T\right)$ where $N_{c}$ and $N_{v}$ are the conduction band and the valence band density of states, respectively, $k_{B} T$ is the thermal energy, and $E_{g}$ is the band gap. We assume a negligible probability for the parasitic absorption in our model. Photons generated by the radiative recombination events, therefore, may either escape from the layer with a probability $P_{\text {esc }}$, or be reabsorbed again. It is assumed that the ratio between the apparent and intrinsic radiative recombination coefficients gives the escape probability as [9]

$$
P_{\mathrm{esc}}=\frac{B_{\mathrm{rad}}^{\prime}}{B_{\mathrm{rad}}}
$$

According to Beer's law, when the layer is illuminated by a collimated beam of photons, an exponential profile of photocarriers is generated through the system. On the other hand, however, radiative recombination of the carriers results in a population of noncollimated (isotropically emitted) photons in the layer. Following Pazos-Outón et al. [3], we model the kinetics of these secondary photons in the layer as a diffusion process with appropriate creation and annihilation terms. Based on this approach, we write the following coupled continuity equations for the carriers and photons:

$$
\begin{gathered}
\frac{\partial \Gamma}{\partial t}=-\frac{\partial j_{\Gamma}}{\partial x}-\beta \Gamma+B_{\mathrm{rad}} n p \\
\frac{\partial n}{\partial t}=-\frac{\partial j_{n}}{\partial x}+I_{0} \alpha e^{-\alpha x}+\beta \Gamma-B_{\mathrm{rad}} n p-\frac{n}{\tau_{\mathrm{SRH}}} \\
-k_{\mathrm{Agr}} n^{2} p .
\end{gathered}
$$

Here, $\Gamma=\Gamma(x, t)$ is the number density of the secondary photons, $j_{\Gamma}=-D_{\Gamma} \partial \Gamma / \partial x$ is the photon current, and $\beta=$ $\alpha c / n_{r}$ is the photon absorption rate constant that is the inverse of the lifetime of the photon. $D_{\Gamma}=c / n_{r} \alpha$ is the photon diffusion coefficient $[3,16,17]$. Note that in contrast to Eq. (1), this is the intrinsic radiative coefficient that appears in Eq. (4), because now the possibility of the PR is explicitly included in the model. To generally analyze the problem, one has to consider the coupling between the populations of the photons and photocarriers through the set of Eq. (4) in combination with appropriate boundary conditions. For the carriers, in the case of a photovoltaic device, for instance, the boundary conditions are straightforwardly determined according to the mode of the device operation.

Setting boundary conditions for the secondary photons is an important step in our modeling because any benefit from the optical design should be introduced via the boundary conditions. Here, for the first time, we propose partially reflecting boundary conditions, as

$$
\begin{gathered}
j_{\Gamma, x=0}=-\mathcal{S}_{f} \Gamma_{x=0} \\
j_{\Gamma, x=d}=\mathcal{S}_{r} \Gamma_{x=d},
\end{gathered}
$$

where velocities $\mathcal{S}_{f}$ and $\mathcal{S}_{r}$ are defined as measures of the strength of the emissivity of the layer at $x=0$ (front surface) and $x=d$ (rear surface), respectively. Note that by setting these boundary conditions, in contrast to Ref. [3], the escape probability will be an output of our approach. These parameters are of great importance in our model because one can take different degrees of photon confinement into account by imposing different escape velocities in the model. Here, we use $\mathcal{S}_{f}=\mathcal{S}_{r}=c / 2 n_{r}$ in our simulations, but from an experimental point of view, one 
can treat these velocities as fitting parameters. Monte Carlo simulation can also be utilized to determine these velocities for a specific optical design [18]. Also note that the quantity $\mathcal{S} \Gamma$ is, in fact, the PL emission of the layer in response to the external radiation. Therefore, in addition to the photocarrier and secondary photon profiles, as an important and valuable output, our formalism can also give the quantitative PL emission from the front $\left(\mathcal{S}_{f} \Gamma_{x=0}\right)$ and rear $\left(\mathcal{S}_{r} \Gamma_{x=d}\right)$ sides of the layer.

Three coupled continuity equations (for $n, p$, and $\Gamma$ ) have to be solved in order to obtain the local carrier and secondary photon densities in the layer. In order to simulate a photovoltaic device, one may also need to consider the Poisson equation. We, however, found that introducing the electric field does not add new physics to our problem. Boundary conditions for the photons are given in Eq. (5). For the carriers, we use the perfect reflecting boundary conditions, as $j_{n, x=0}=j_{n, x=d}=0$ for the electrons, and similarly for the holes. These conditions imply that at the steady state, the carrier density can, with a good approximation, be considered uniform. Therefore, in the following, we use the average carrier densities to calculate the PL internal and external efficiencies. For the transient conditions in Secs. III B and III C, we also use the average values to obtain the various internal efficiencies. (While the local efficiencies can also be calculated, they are not common in the literature. However, we confirm that although the local efficiencies exhibit more complex dynamics, the trends obtained using the average densities still remain valid). As we discuss in section "Results and Discussion," there are two methods to calculate the apparent radiative coefficient, $B_{\text {rad }}^{\prime}$. In the traditional method, one uses the continuity Eq. (1) to estimate $B_{\text {rad }}^{\prime}$. To do this, we first solve coupled Eq. (4) to obtain the true densities $n$ and $p$. Then we find $B_{\text {rad }}^{\prime}$ in a way that the same average densities are established in the system.

As we mention in the beginning of this section, here we model the PL dynamics for the illumination by photons with energy near the absorption edge of the layer. In the experiment, however, the layer is usually illuminated by a higher-than-band-gap energy photon flux (to ensure a high absorption), and one also deals with a spectral PL emission from the layer $[2,6,7]$. In fact, time-resolved PL spectra can give valuable information about the PR efficiency of the layer. Extension of Eq. (4) to model the PL spectra is straightforward. To this end, one needs to write the continuity equation for a series of the photon population, as (see Ref. [3] and supporting information of Ref. [16])

$$
\frac{\partial \Gamma_{i}}{\partial t}=-\frac{\partial j_{\Gamma_{i}}}{\partial x}-\beta_{i} \Gamma_{i}+P\left(E_{i}\right) B_{\mathrm{rad}} n p
$$

where $P\left(E_{i}\right) \propto \exp \left[-\left(E_{i}-E_{0}\right)^{2} / 2 \sigma^{2}\right]$ is the probability that a photon with energy $E_{i}$ is emitted in a radiative recombination event. $E_{0}$ is the peak of the PL spectrum
TABLE I. Constants used in the modeling.

\begin{tabular}{ll}
\hline \hline$D_{n}$ & $1.7 \times 10^{-2} \mathrm{~cm}^{2} \mathrm{~s}^{-1}$ \\
$D_{p}$ & $1.1 \times 10^{-2} \mathrm{~cm}^{2} \mathrm{~s}^{-1}$ \\
$N_{c}$ & $10^{19} \mathrm{~cm}^{-3}$ \\
$N_{v}$ & $10^{19} \mathrm{~cm}^{-3}$ \\
$k_{B} T$ & $0.026 \mathrm{eV}$ \\
$E_{g}$ & $1.61 \mathrm{eV}$ \\
$\alpha$ & $5 \mu \mathrm{m}^{-1}(\mathrm{see}$ also Fig. 7) \\
$c$ & $3 \times 10^{8} \mathrm{~ms}^{-1}$ \\
$n_{r}$ & 2.5 \\
$B_{\mathrm{rad}}$ & $5 \times 10^{-10} \mathrm{~cm}^{3} \mathrm{~s}^{-1}$ \\
$1 / \tau_{\mathrm{SRH}}$ & $3 \times 10^{6} \mathrm{~s}^{-1}$ \\
$k_{\mathrm{Agr}}$ & $10^{-29} \mathrm{~cm}^{6} \mathrm{~s}^{-1}$ (see also Fig. 5) \\
\hline \hline
\end{tabular}

in the absence of the PR effect and $\sigma \sim 0.1 \mathrm{eV}$. In the following sections, we discuss our results for the solution of Eq. (4) for both the steady-state and transient conditions. Parameter values used in our simulation can be found in Table I.

\section{RESULTS AND DISCUSSION}

\section{A. Steady-state conditions}

To examine whether the model can explain the experimental observations discussed in the introductory section, we first study the internal and external PL quantum efficiencies of the layer under steady-state illumination conditions. By solving the coupled Eq. (4), we are able to calculate both the internal and external quantum efficiencies of the layer, as

$$
\eta_{\text {int }}=\frac{B_{\mathrm{rad}} n p}{B_{\mathrm{rad}} n p+\left(n / \tau_{\mathrm{SRH}}\right)+k_{\mathrm{Agr}} n^{2} p}
$$

and

$$
\eta_{\mathrm{ext}}=\frac{\mathcal{S}_{f} \Gamma_{x=0}+\mathcal{S}_{r} \Gamma_{x=d}}{I_{0}\left(1-e^{-\alpha d}\right)} \equiv \eta_{\mathrm{ext}, \mathrm{f}}+\eta_{\mathrm{ext}, \mathrm{r}}
$$

Then, since the two efficiencies are generally related to each other as below, we can find the escape probability $P_{\text {esc }}$ from our model. In general [9],

$$
\eta_{\mathrm{ext}}=\frac{\eta_{\mathrm{int}} P_{\mathrm{esc}}}{1-\eta_{\mathrm{int}}+\eta_{\mathrm{int}} P_{\mathrm{esc}}}
$$

A completely different method to determine the escape probability is based on the continuity Eq. (1) and the definition given in Eq. (3). In this approach, we fit Eq. (1) to the true solution already obtained from the coupled Eq. (4), thereby we can find coefficient $B_{\text {rad }}^{\prime}$ that can recover the true solution. In other words, we consider the system with the PR effect and the intrinsic recombination coefficient, but the results are explained using an apparent radiative recombination coefficient. This method has been used to obtain the apparent radiative lifetime 


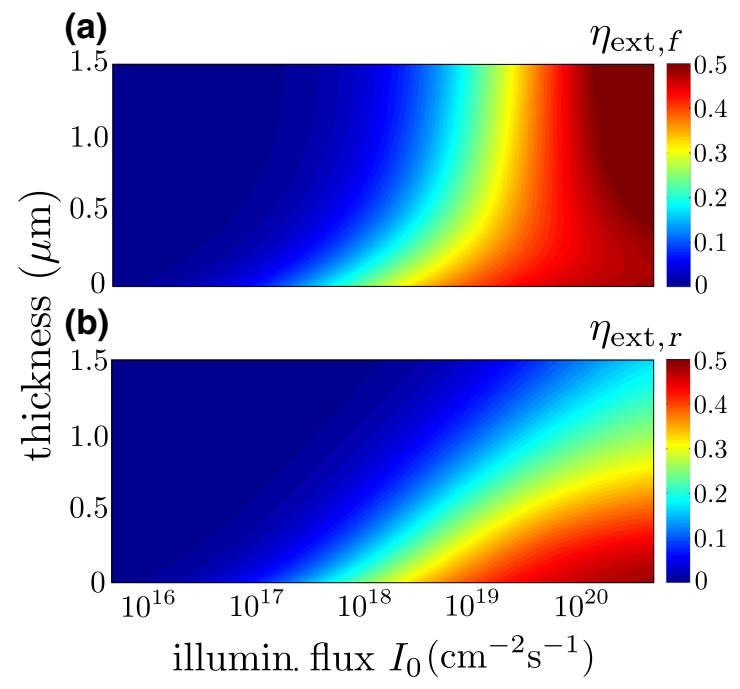

FIG. 1. Mapping of the PL quantum efficiency for the front (a), and rear (b) surfaces of the layer, for a wide range of the illumination flux $I_{0}$ and film thickness $d$, under steady-state illumination conditions.

$\left(\propto 1 / B_{\mathrm{rad}}^{\prime}\right)$ in the experiments where the model Eq. (1) is fitted to the results obtained from the experimental measurements $[8,10]$. For a wide range of the illumination flux $\left(I_{0}\right)$ and film thicknesses $(d)$, we find that both methods result in approximately the same escape probabilities; see Supplemental Material [19] for detailed results. This is an important result that indicates the consistency of our formalism. In the following, we use the first method to obtain the escape probability, and then using Eq. (3), we find $B_{\text {rad. }}^{\prime}$.

Figure 1 shows the map of normalized PL emission from the front $\left(\eta_{\text {ext, f }}\right)$ and rear $\left(\eta_{\text {ext, }, \mathrm{r}}\right)$ surfaces of the layer. In both cases, by increasing the illumination flux intensity, the PL efficiency increases. This is because at high intensities, since the density of the photocarrier in the layer increases $\left(n \sim 10^{17} \mathrm{~cm}^{-3}\right)$, the radiative channel becomes the dominant recombination loss in the layer, and therefore, the layer shows a high internal quantum efficiency $\left(\eta_{\text {int }} \approx 0.8\right)$; see Supplemental Material [19] for the map of charge density and internal efficiency. On the other hand, the front and rear PLs show different behaviors with respect to the thickness of the layer. By increasing the thickness, in contrast to the front side, the PL efficiency from the rear side decreases because, owing to the nonradiative recombination channel, the chance for the carriers and radiatively generated photons to reach the rear side decreases (as in the limit of a semi-infinite layer, the PL from the rear side is expected to vanish).

As discussed before, one of the major observations related to the PR effect in the perovskite layers is that a change in the light out-coupling of the system alters its measured (apparent) radiative recombination coefficient.

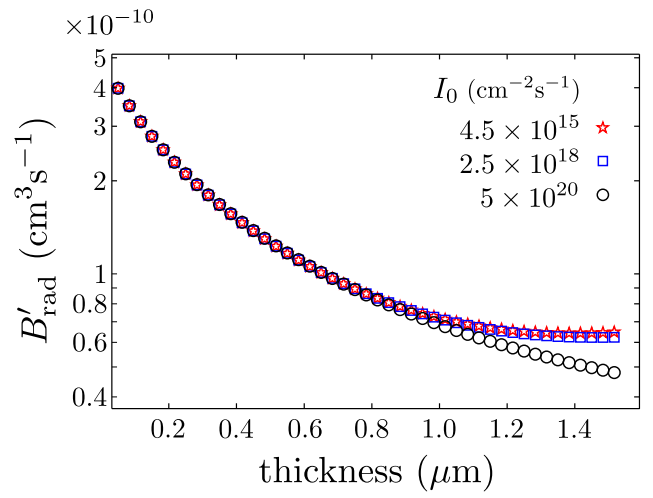

FIG. 2. Apparent radiative recombination coefficient vs layer thickness for three different illumination fluxes under steadystate conditions.

In our model, we can study the effects of different light out-couplings via changing the film thickness and (or) the velocities $\mathcal{S}_{f}$ and $\mathcal{S}_{r}$. Figure 2 shows our results for $B_{\text {rad }}^{\prime}$ as a function of the film thicknesses for three different illumination fluxes. In agreement with the experimental observations $[8,10]$, the apparent recombination coefficient decreases from the intrinsic value (here, $B_{\text {rad }}=$ $5.0 \times 10^{-10} \mathrm{~cm}^{3} \mathrm{~s}^{-1}$ ) upon increasing the thickness. This is because the higher the thickness of the layer, the lower the chance for a photon to escape from the layer before being reabsorbed again. Consequently, a radiative recombination event may not really give rise to a loss of the carriers, leading to a higher apparent lifetime. Also, as can be seen in Fig. 2, the behavior is independent of the flux intensity (except for $d \geq 1.2 \mu \mathrm{m}$ ), suggesting that in the steady-state conditions, the escape probability is a welldefined parameter of the system, independent of external perturbations. We revisit this issue in section $\mathrm{C}$ where we show that the escape probability is time-dependent in some transient conditions, and therefore, cannot be considered as a constant in the corresponding problems.

\section{B. Transient conditions}

Now, we consider the time-dependent problem in which the response of the system to a transient excitation (a laser pulse, for example, as in the transient spectroscopy or timeresolved PL) is explored by the model. To simulate the excitation pulse, instead of $I_{0}$ we use a Gaussian generation term in Eq. 4(b), as

$$
\frac{I_{\mathrm{pul}}}{\sqrt{2 \pi \sigma_{t}^{2}}} \exp \left(-\frac{t^{2}}{2 \sigma_{t}^{2}}\right)
$$

where $\sigma_{t}$ is the pulse temporal width and $I_{\text {pul }}$ is its total photon fluence. (In the experiments, the total energy fluence is usually reported, which can easily be converted to the total photon fluence. For example, a $4 \mu \mathrm{J} / \mathrm{cm}^{2}$ energy fluence of photons with an energy of $1.5 \mathrm{eV}$ is equivalent to 


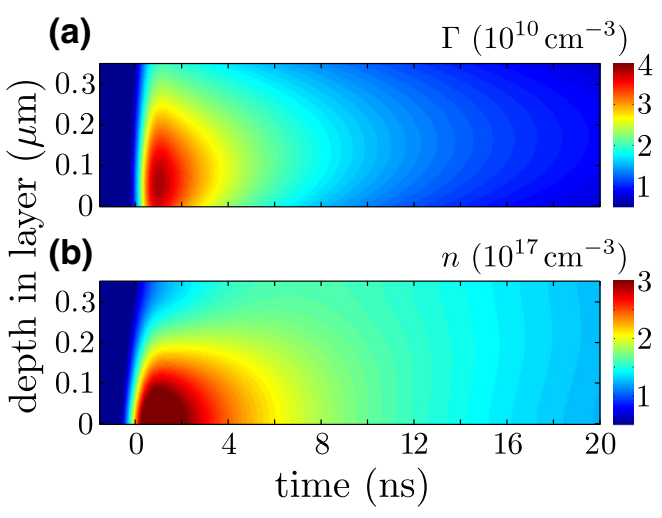

(c)

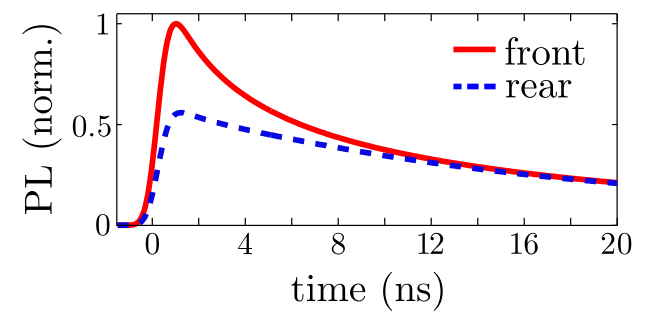

FIG. 3. Time evolution of secondary photon (a), and carrier local densities (b) for a layer with $d=0.35 \mu \mathrm{m}$, in response to the excitation pulse with fluence $I_{\mathrm{pul}}=10^{13}$ photon $/ \mathrm{cm}^{2}$. Panel (c) shows the corresponding normalized PL emission from the front $(x=0)$ and rear sides $(x=d)$ of the layer.

$I_{\text {pul }} \approx 1.7 \times 10^{13}$ photon $/ \mathrm{cm}^{2}$.) We use $\sigma_{t}=0.5 \mathrm{~ns}$ in our simulations.

The evolution of the local density of the carriers and secondary (radiatively generated) photons following the excitation pulse are illustrated in Fig. 3 for a layer of $d=0.35 \mu \mathrm{m}$. Let us first look at the charge density profile, Fig. 3(b). According to Beer's law, as time goes on, an exponential profile of the carriers is generated in the system. Therefore, the smaller the depth in the layer, the higher the local charge density $n$, as can be seen in the figure. However, after time $t \approx 2 \sigma_{t}=1 \mathrm{~ns}$ where the excitation pulse finishes, the spatial distribution of the carriers tends to be uniform and, as seen, a uniform distribution is reached at time $t \approx 10 \mathrm{~ns}$. There are two mechanisms that make this happen: the diffusion process, by which the carriers spread in the film, and the PR effect, which, even in the absence of the diffusion process, spreads the carriers over a distance of approximately $\alpha^{-1}$ in each recycling event. The role of each of these effects is addressed in the following and also in Supplemental Material. In summary, we find that at high excitation fluences (or, equivalently, high carrier densities of approximately $10^{18} \mathrm{~cm}^{-3}$ ) the PR effect plays the dominant role. Instead, at low fluences (approximately $10^{15} \mathrm{~cm}^{-3}$ ) the diffusion process becomes the determining factor. Our result, obtained based on the photon diffusion concept, is also in agreement with the picture provided by Crothers et al. who studied the PR effect using the ray-tracing method [8].

As seen in Fig. 3(a), the density of the secondary photons has its maximum at depth $x \approx 100 \mathrm{~nm}$ (and not $x=$ 0 ), which is the typical radiative diffusion length at densities $10^{17}-10^{18} \mathrm{~cm}^{-3}$. The photon distribution becomes symmetric at time $t \approx 10 \mathrm{~ns}$ owing to the mechanisms described above, and also because we have chosen the same velocities $\mathcal{S}$ for the rear and front surfaces. As a result of this symmetric distribution, the front and rear PLs are approximately equal at the mentioned time, as seen in Fig. 3(c). Furthermore, since the generated photons can escape from the system, the layer is somewhat more depleted of photons at the boundaries.

The effect of the excitation fluence on the PL dynamics in the halide perovskite films has recently been experimentally investigated $[4,6]$. A common observation is that the PL decay is greatly influenced by the fluence intensity and at high excitation fluences, the decay exhibits a nonexponential behavior. We note that the PL intensity is proportional to $n p \approx n^{2}$, and therefore, one can write

$$
\frac{d}{d t} \mathrm{PL} \propto 2 n \frac{d n}{d t}
$$

Furthermore, in the low-density regime, if we neglect the carrier diffusion, we can write $d n / d t \approx-n / \tau_{\mathrm{SRH}}$, and therefore, an exponential decay is expected, as

$$
\mathrm{PL} \propto e^{-\left(2 / \tau_{\mathrm{SRH}}\right) t}
$$

Figure 4(a) shows our simulation results for the front PL under three different excitation fluences. These results are in agreement with the experimental observations discussed above. Let us first consider the low-fluence case, curve (i). Except at short times, the decay is exponential and can be explained well by Eq. (11), meaning that the monomolecular recombination mechanism is the dominant loss channel at this fluence. As a result, the PL decay in the low excitation conditions can safely be used to determine the monomolecular nonradiative lifetime, $\tau_{\mathrm{SRH}}$. An initial nonexponential decay component can also be seen in curve (i). In Fig. 4(b), a simulated PL decay for the same excitation fluence but with $D_{n}=D_{p}=0$ is shown. The result clearly shows that the nonexponential part results from the photocarriers' diffusion that decreases the initial local photocarrier density, leading to a fast decrease in the PL intensity. We also conclude that the PR does not have a significant effect on the decay dynamics at low fluences. That is why a simple diffusion equation without the PR term can reproduce the experimental measurements [20].

In contrast to the low excitation fluence, at high fluences, the decay is purely nonexponential in the time range considered here, curves (ii) and (iii) in Fig. 4(a). In fact, owing to the high photocarrier density created at these excitation 

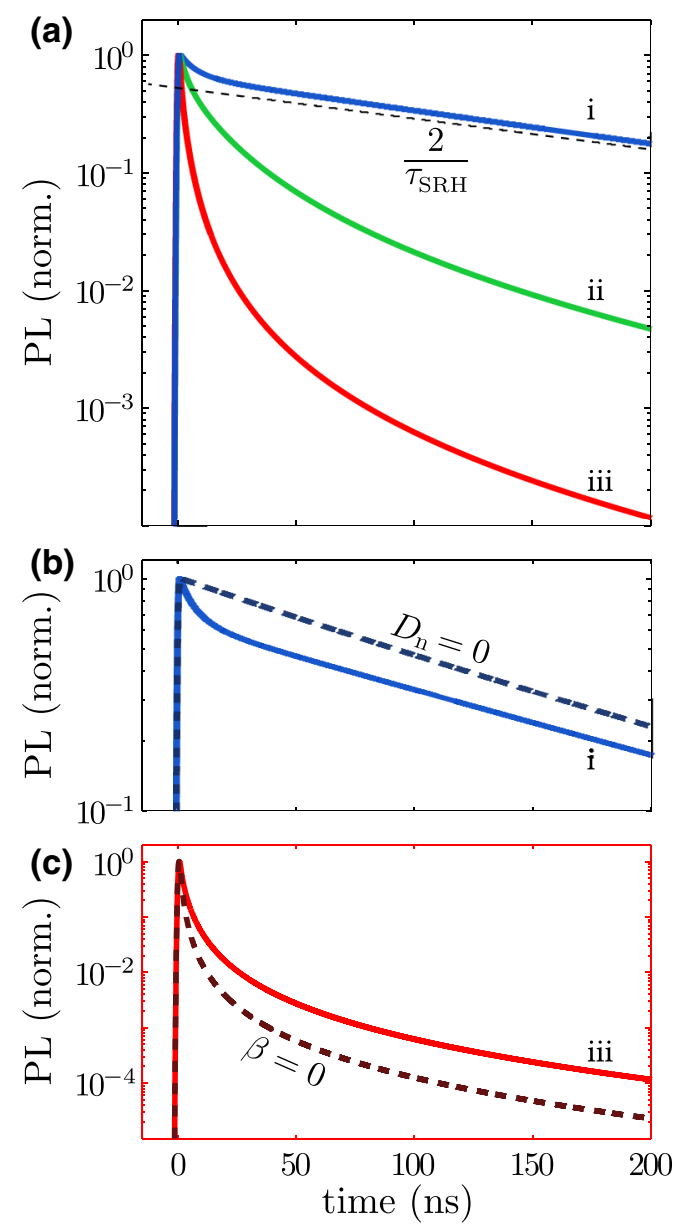

FIG. 4. (a) Normalized PL emission from the front side for a layer with $d=0.35 \mu \mathrm{m}$, under excitation fluences of (i) $10^{11}$, (ii) $10^{13}$, and (iii) $10^{14}$ photon $/ \mathrm{cm}^{2}$. Dashed line corresponds to an exponential decay with decay rate $2 / \tau_{\text {SRH }}$. (b) Effect of the carrier diffusion on the decay dynamics at low excitation fluence. Curve (i) is the same as in part (a), but dashed curve shows the decay when $D_{n}=D_{p}=0$. (c) Effect of the PR on the decay dynamic at high excitation fluence. Curve (iii) is the same as in part (a). Dashed curve shows the decay in the absence of the PR possibility, that is, simulation with $\beta=0$ in Eq. (4).

fluences, the dominant process in the layer is the bimolecular radiative recombination of the carriers. We also find that the carrier diffusion becomes totally irrelevant. As a result of the efficient radiative recombination, the PR effect is expected to be significant. Figure 4(c) shows the PL decays with and without considering the PR effect [by imposing $\beta=0$ in Eq. (4), we can simulate the response of the layer in the absence of the PR possibility]. As seen, without the PR possibility, the decay is faster because the photocarriers lost by the radiative recombination do not have any chance to be created again. In other words, in this case, the carrier population is reduced by the intrinsic rate coefficient $B_{\text {rad }}$ rather than $B_{\text {rad }}^{\prime}$. Recently, time-resolved PL spectra of the single crystal perovskites has revealed a subtler interplay between the PR efficiency, the diffusion process, and the nonradiative recombination lifetime [21,22]: in the layers with a long nonradiative lifetime, the carriers can diffuse deep in the layer, and consequently, the photons created by radiative recombination of these carriers may be reabsorbed again. Since the higher the energy of the photons, the stronger the reabsorption, a redshift in the PL spectrum is observed.

The radiative recombination channel dominates over the Shockley-Read-Hall mechanism at high excitation fluences, leading to an increase in the internal quantum efficiency $\eta_{\text {int }}$. However, there is still an efficient nonradiative process at these conditions; the Auger recombination. Solid lines in Fig. 5(a) show $\eta_{\text {int }}$ as a function of time for two different Auger rate constants. As seen in the figure, there is a drop in $\eta_{\text {int }}$ at initial times, which is then recovered as time goes on. In Fig. 5(b), we plot Auger recombination efficiency $\eta_{\text {Agr }}$ (fraction of Auger to the total recombination rate),

$$
\eta_{\mathrm{Agr}}=\frac{k_{\mathrm{Agr}} n^{2} p}{B_{\mathrm{rad}} n p+\left(n / \tau_{\mathrm{SRH}}\right)+k_{\mathrm{Agr}} n^{2} p}
$$

which clearly shows that the reason for the drop in $\eta_{\text {int }}$ in (a) is the Auger recombination channel. At later times, however, owing to the decrease in the carrier density through different recombination mechanisms, $\eta_{\text {Agr }}$ declines, and therefore, a high internal quantum efficiency is re-established. Figure 5 also shows results of our simulations with $\beta=0$, that is, without considering the PR
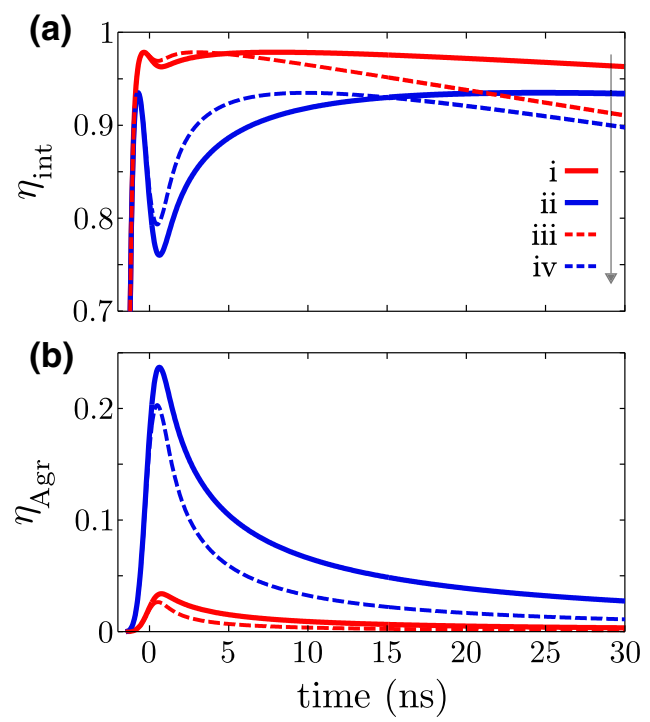

FIG. 5. (a) Internal quantum efficiency $\eta_{\text {int }}$ and (b) Auger recombination efficiency $\eta_{\text {Agr }}$ for a layer of $d=0.35 \mu \mathrm{m}$, with (i) $k_{\mathrm{Agr}}=10^{-29}$ and (ii) $10^{-28} \mathrm{~cm}^{6} \mathrm{~s}^{-1}$, in response to the excitation fluence $I_{\text {pul }}=10^{14}$ photon $/ \mathrm{cm}^{2}$. Dashed lines, curves (iii) and (iv) in both panels, show the corresponding results without the PR possibility, that is, for simulations with $\beta=0$. 


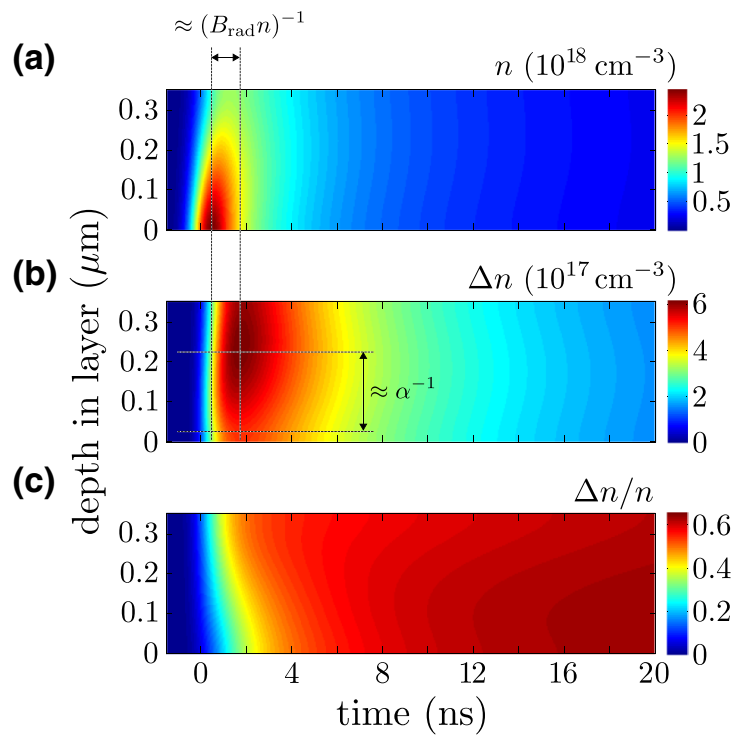

FIG. 6. (a) Time evolution of the carrier local densities for a layer with $d=0.35 \mu \mathrm{m}$, in response to the excitation fluence $I_{\mathrm{pul}}=10^{14}$ photon $/ \mathrm{cm}^{2}$. (b) Dynamics of the quantity $\Delta n=n-$ $n_{\text {noPR }}$. Here, $n$ is the density shown in part (a), and $n_{\text {noPR }}$ is the density obtained from Eq. (4) with $\beta=0$, that is, without the PR term. High value regions in parts (a) and (b) are separated from each other by $\Delta x \approx \alpha^{-1}$ and $\Delta t \approx\left(B_{\mathrm{rad}} n\right)^{-1}$ with $n \approx 2 \times 10^{18} \mathrm{~cm}^{-3}$. (c) Time evolution of the quantity $\Delta n / n$.

possibility in the layer (dashed lines in the figure). Interestingly, in the absence of the PR effect, the layer shows a higher internal efficiency at initial times. This is because without the PR possibility the carrier density decreases faster, which in turn, leads to a lower Auger recombination, as seen from dashed lines in Fig. 5(b). As a result, the PR effect may increase the high-order nonradiative recombination efficiency at high densities.

In order to further assess the effect of the PR on the carrier dynamics, let us see how the PR affects the density evolution in the layer. Figure 6(a) shows the carrier dynamics across the film depth in response to the excitation fluence of $I_{\text {pul }}=10^{14}$ photon $/ \mathrm{cm}^{2}$ [with the PL emission already shown in Fig. 4, curve (iii)]. Note that the highest density established in the layer is approximately equal to $2 \times 10^{18} \mathrm{~cm}^{-3}$, implying a radiative lifetime of $\left(B_{\mathrm{rad}} n\right)^{-1} \approx 1 \mathrm{~ns}$. In addition, the photons resulting from the radiative recombination are expected to be absorbed after traveling a distance $\alpha^{-1}=0.2 \mu \mathrm{m}$. On the other hand, without considering the PR possibility in the model, we get a lower density from the simulation; let us call it $n_{\text {noPR }}$. The difference between the two simulations, $\Delta n=n-n_{\text {noPR }}$, is shown in Fig. 6(b). Interestingly, the largest difference between the two densities is found to be about $\Delta t \approx 1 \mathrm{~ns}$ after and $\Delta x \approx 0.2 \mu \mathrm{m}$ away from the region where the photocarrier density is maximum. This obviously shows how the PR effect can spread the photocarrier in the layer much faster than that expected from the diffusive motion. It is clear that as time goes on, the role of the PR becomes more pronounced, as can be seen in Fig. 6(c) where the relative difference $\Delta n / n=1-n_{\text {noPR }} / n$ is illustrated.

\section{Escape probability in the transient conditions}

As we discuss in Secs. II and III A, $P_{\text {esc }}$ is the probability that an emitted photon in the interior region of the layer escapes to the outside. It should be noted that although the secondary photons are emitted at different locations inside the layer, in the steady-state conditions, there is an effective escape probability so that once the escape probability is obtained, it can be used for any photon wherever it is emitted inside the layer. In fact, in the steady state, a combination of the optical design of the layer in its interfaces and the thickness of the layer uniquely determines $P_{\text {esc }}$. Therefore, as we state in section A, the escape probability can be considered a well-defined parameter of the layer, independent of the external illumination. In Supplemental Material we show the escape probability for a wide range of the illumination flux and film thickness $d$ under steady-state illumination conditions. In the following, we examine the validity of a constant well-defined $P_{\text {esc }}$ in the transient conditions. We see that because of the dynamical anisotropy in the density of the secondary photons, the escape probability shows a transient behavior before reaching its steady-state value at longer times.

We note that the two different methods described in Sec. III A to determine $P_{\text {esc }}$, one based on Eq. (3) and the other based on Eq. (9), are appropriate principally for the steadystate conditions. We propose a more fundamental way to obtain the escape probability, appropriate in the transient conditions, by calculating the ratio of the photons emitted from the layer at times $\leq t$ (PL emission of the layer) to the total photons generated inside the layer (as the result of the radiative recombination), as

$$
P_{\mathrm{esc}}(t)=\frac{\int_{t_{0}}^{t} \operatorname{PL}\left(t^{\prime}\right) d t^{\prime}}{\int_{t_{0}}^{t} \int_{0}^{d} U_{\mathrm{rad}}\left(x, t^{\prime}\right) d x d t^{\prime}}
$$

Here, $\operatorname{PL}\left(t^{\prime}\right)$ is the photoluminescence emission from the front and rear sides of the layer at time $t^{\prime}$, given by Eq. (5), and $U_{\mathrm{rad}}\left(x, t^{\prime}\right)=B_{\mathrm{rad}} n\left(x, t^{\prime}\right) p\left(x, t^{\prime}\right)$ is the radiative recombination rate in the layer at time $t^{\prime}$ and position $x$. Also, $t_{0}$ is the time that the laser pulse is applied [practically, approximately $-2 \sigma_{t}$; see Eq. (10)]. It should be noted that one can also use Eq. (9) in order to obtain the escape probability in the transient conditions. However, this method results in an artificial time-dependent behavior for $P_{\text {esc }}$. This is because the secondary photons are generated inside the layer after the time of approximately $\left(B_{\mathrm{rad}} n\right)^{-1}$ and this nonrelevant timescale enters into the problem via the parameter $\eta_{\text {ext }}$ in Eq. (9). Finally, we note that as a result 

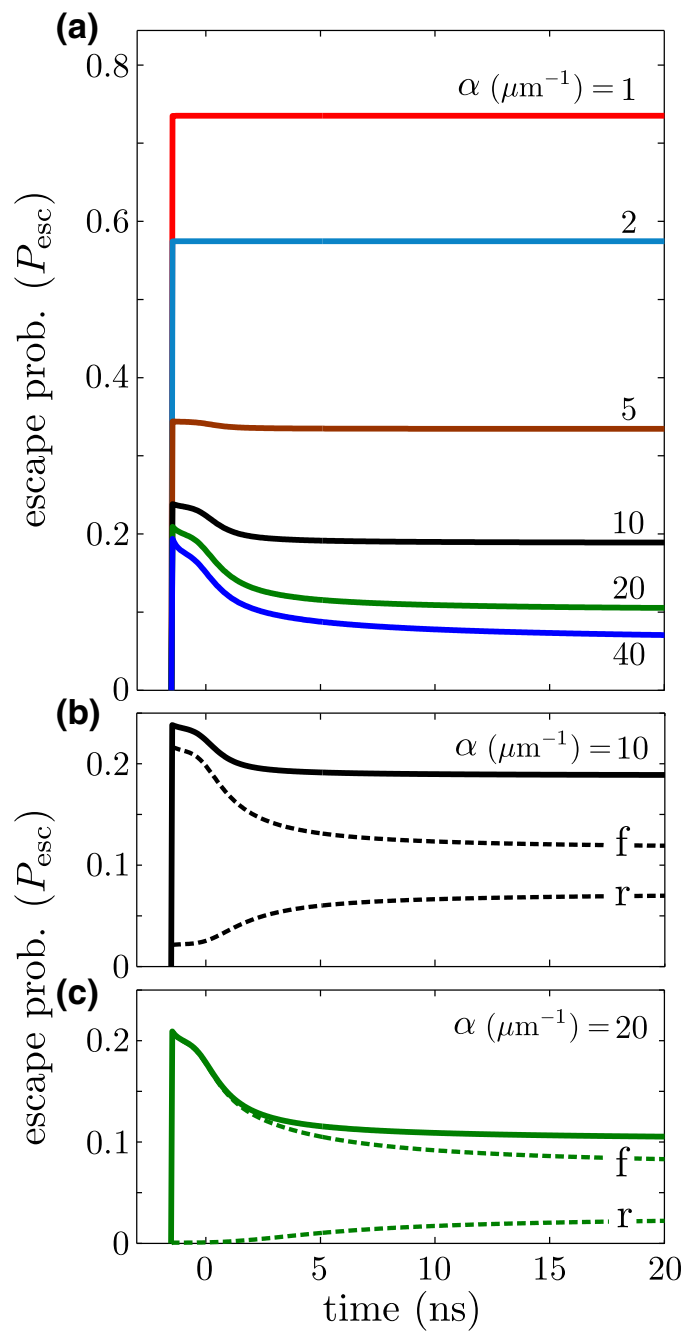

FIG. 7. (a) Escape probability as a function of time for the layers with $d=0.35 \mu \mathrm{m}$ and different absorption coefficient $\alpha$, in response to the excitation fluence $I_{\text {pul }}=10^{14}$ photon $/ \mathrm{cm}^{2} . P_{\text {esc }}$ for the cases $\alpha=20$ and $40 \mu \mathrm{m}^{-1}$ reaches its stationary value at times of approximately $50 \mathrm{~ns}$, not shown here. (b),(c) Show the escape probability from the front (f) and rear (r) sides of the layer for two different $\alpha$. Solid curves in (b) and (c) are the sum of their corresponding dashed curves.

of the PL emission from both the front and rear sides of the layer, $P_{\text {esc }}$ in Eq. (14) can be separated into two probabilities, as $P_{\text {esc }}(t)=P_{\text {esc }, \mathrm{f}}(t)+P_{\text {esc, } \mathrm{r}}(t)$.

Figure 7 shows the escape probability as a function of time for a layer of thickness $d=0.35 \mu \mathrm{m}$ in response to the excitation fluence of $I_{\text {pul }}=10^{14}$ photon $/ \mathrm{cm}^{2}$, calculated using Eq. (14). As discussed in Fig. 6, the PR effect becomes an important process at this intensity, enabling us to efficiently study the escape probability. In Fig. 7(a), $P_{\text {esc }}$ is shown for layers with different absorption coefficient values. First, it can be seen that in all cases, the escape probabilities reach stationary values at sufficiently long times. These stationary values are, in fact, the same as one obtains under steady-state illumination conditions (for example, the result for $\alpha=5 \mu \mathrm{m}^{-1}$ is the same as that obtained from figure 1 in Supplemental Material). For short times on the other hand, the behavior of the escape probability depends on the absorption coefficient value: while for small $\alpha$ (that is, $\alpha d<1$ ) $P_{\text {esc }}$ is time independent, the results for large $\alpha(\alpha d>1)$ show a clear transient behavior so that at short times, $P_{\text {esc }}$ is higher than its stationary value. In order to explain the results in Fig. 7(a), we note that a large absorption coefficient implies a very initial inhomogeneous density of photons in the layer. Since most of the secondary photons are initially emitted near the front side, the probability that the photons can leave the layer from the front side is high. Although for the same reason the escape probability is low from the opposite side, the behavior of $P_{\text {esc }}$ is mainly determined by the front side because the photons are concentrated near this surface. This is clearly seen in Figs. 7(b) and 7(c), where the escape probabilities from the front and rear sides of the layer are presented for $\alpha=10$ and $20 \mu \mathrm{m}^{-1}$. As time goes on, the photons are redistributed in the layer as a result of the PR effect, leading to a decrease in the escape probability. However, it should be noted that the anisotropy between the front and rear sides does not disappear completely even at long times.

As pointed out in Sec. II, an alternative way for modeling the PR effect, used in Ref. [3], is to use $P_{\text {esc }}$ as an input of the model by incorporating it directly (as a constant) into the continuity equation for the photons, as

$$
\frac{\partial \Gamma}{\partial t}=-\frac{\partial j_{\Gamma}}{\partial x}-\beta \Gamma+\left(1-P_{\mathrm{esc}}\right) B_{\mathrm{rad}} n p
$$

In this case, instead of the boundary conditions introduced in Eq. (5), one should use a completely reflecting boundary condition for the photons. With the stationary values obtained for $P_{\text {esc }}$ in Fig. 7, we also obtain the photon density using the above continuity equation and compare it to the correct solution based on our model given by Eq. (4); see Supplemental Material [19] for the results and more details where it is shown that the two approaches differ significantly for the cases where $P_{\text {esc }}$ is time dependent. Therefore, from the results presented in Fig. 7 and Supplemental Material [19], we conclude that describing a layer with a constant (effective) escape probability is not correct for the time-dependent problems. From the experimental point of view, the results in Fig. 7 suggest that a progressive redshift of the transient PL spectra should be observed with time in the perovskite layers. In fact, because according to Fig. 7, the probability of the escape of a high-energy photon (corresponding to a high $\alpha$ ) decreases with time, the PL spectra should show an increasing redshift with time on the timescale of approximately $20 \mathrm{~ns}$. These results are indeed in complete agreement with the experimental observation reported by Yamada et al. [21]. 


\section{Effective diffusion length}

In the early work by Stranks et al. [23], it was found that the carrier diffusion length $L_{D}$ in the perovskite absorbers can be as large as $1 \mu \mathrm{m}$. This important result was obtained by transient photoluminescence measurements with pulse energy fluences of approximately $10 \mathrm{~nJ} / \mathrm{cm}^{2}$. As we discuss above, the dominant recombination pathway at these excitation energies is the first-order nonradiative loss. At higher fluences, however, as a result of the efficient radiative recombination and possible subsequent PR event, the radiatively recombined carriers have a chance to be retrieved again. Therefore, the effective diffusion length $L_{\text {eff }}$ (the length that a carrier travels before being lost by a nonradiative recombination event) can be larger than that traditionally defined as $L_{D}=\sqrt{D \tau}$ with $\tau$ being the intrinsic lifetime of the carriers,

$$
\tau=\frac{1}{1 / \tau_{\mathrm{SRH}}+B_{\mathrm{rad}} n+k_{\mathrm{Agr}} n^{2}}
$$

Here, we give an expression for the effective diffusion length in the steady-state conditions in which the PR effect is also taken into account. To do that, we write the total displacement for a carrier as

$\vec{L}=\vec{L}_{D}+\Lambda \vec{L}_{\mathrm{PR}}+\Lambda \vec{L}_{D}^{\prime}+\Lambda^{2} \vec{L}_{\mathrm{PR}}^{\prime}+\Lambda^{2} \vec{L}_{D}^{\prime \prime}+\Lambda^{3} \vec{L}_{\mathrm{PR}}^{\prime \prime}+\cdots$

where $\Lambda=\eta_{\text {int }}\left(1-P_{\text {esc }}\right)$ and $L_{\mathrm{PR}} \sim \alpha^{-1}$. In writing Eq. (17), we use the fact that by each PR event (that takes place with a probability of $\Lambda$ ), the carrier is virtually transferred over a distance of approximately $\alpha^{-1}$ in the layer; see also Fig. 8. Also note that as seen from figure 1 in Supplemental Material, $P_{\text {esc }} \approx 0$ for thick layers (that is, $\Lambda \approx \eta_{\text {int }}$ ), and also $P_{\text {esc }} \approx 1$ for thin layers. We define the effective diffusion length as $L_{\text {eff }}=\sqrt{\left\langle L^{2}\right\rangle}$, where \langle\rangle denotes statistical averaging. As we show in more detail in the APPENDIX, since the secondary photons are isotropically emitted, we can do the averaging and get

$$
L_{\mathrm{eff}}=\sqrt{\frac{D \tau+\Lambda^{2} / \alpha^{2}}{1-\Lambda^{2}}} .
$$

This important result quantitatively shows how the efficiency of the radiative recombination and optical design of the system can change the effective length that carriers can travel in the layer. We note that in the case of $\eta_{\text {int }} \approx 0$, or $P_{\text {esc }}=1$, Eq. (18) is reduced to the traditional definition. Also, note that $L_{\text {eff }}$ is not simply given by $\sqrt{D \tau^{\prime}}$, where $\tau^{\prime}$ is the apparent lifetime of the carriers. This is because the PR effect does not merely change the apparent lifetime, but also transfers the carriers over a distance $\alpha^{-1}$ at each recycling event.

Figure 9 shows $L_{\text {eff }}$, calculated using Eq. (18), as a function of the illumination flux for two different

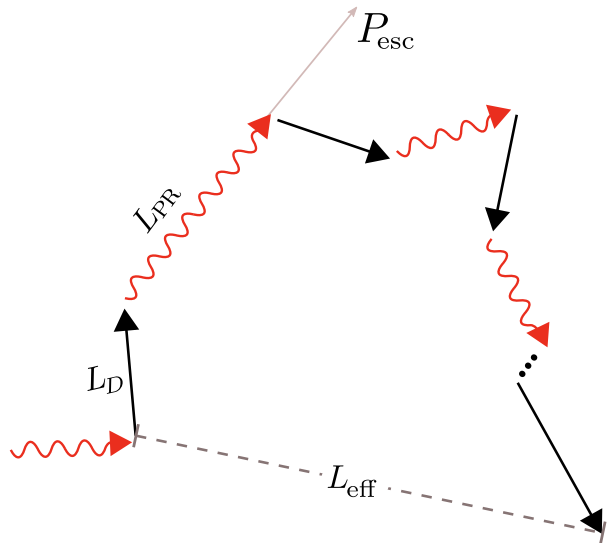

FIG. 8. Schematic illustration showing how the PR effect can increase the effective diffusion length of the carriers in the layer. A carrier after being created by an absorbed photon diffuses in the layer over a distance $L_{D} \sim \sqrt{D \tau}$. In the case of the radiative recombination (occurring with the probability of $\eta_{\text {int }}$ ), a photon is isotropically emitted. This secondary photon may either escape from the layer (with the probability of $P_{\text {esc }}$ ), or may reabsorbed again, and therefore, the recombined carriers are recreated. Absorption length of the secondary photon is $L_{\mathrm{PR}} \sim$ $\alpha^{-1}$. Effective diffusion length is the average distance to the point where a nonradiative recombination occurs and the carriers are lost.

layer thicknesses. For comparison, the traditional diffusion length $L_{D}$ is also presented. At low illumination flux, irrespective of the thickness, $L_{\mathrm{eff}}=L_{D}=\sqrt{D \tau_{\mathrm{SRH}}} \approx 0.8 \mu \mathrm{m}$. This is because at these fluxes $\eta_{\text {int }} \approx 0$ and $\tau \approx \tau_{\mathrm{SRH}}$. This result is in agreement with the experimental conclusion reached by Fang et al. [11] that at low illumination flux (1 Sun, for example), the long carrier diffusion length originates from a long $\tau_{\mathrm{SRH}}$ and not the PR effect. By increasing the flux intensity, however, since the carrier density increases in the layer, the carrier lifetime decreases, leading to a decrease in the transport length. But by

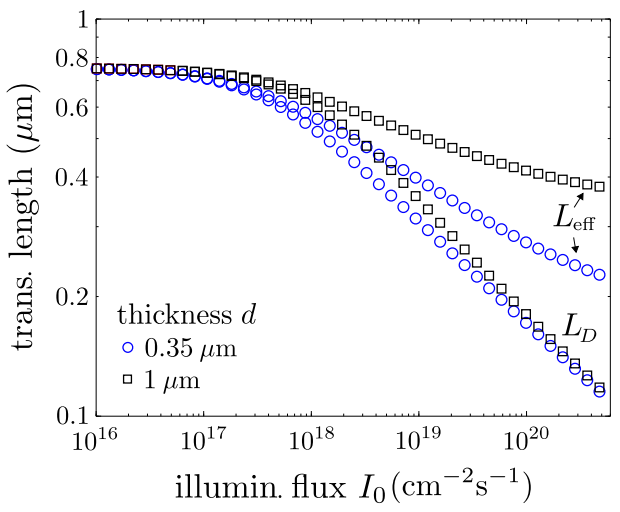

FIG. 9. Transport length (traditional diffusion length $L_{D}$ and effective diffusion length $L_{\text {eff }}$ ) as a function of the illumination flux $I_{0}$, under steady-state conditions. Results are for thickness layers of $d=0.35$ and $1 \mu \mathrm{m}$. 
increasing the charge density, the radiative recombination channel also increases, and hence the PR effect becomes probable. As a consequence, at moderate and high illumination fluxes, we have $L_{\mathrm{eff}}>L_{D}$. Note that the escape probability $P_{\text {esc }}$ is a function of the layer thickness (the thicker the layer, the smaller the escape probability; see Supplemental Material [19] for a complete map of $P_{\text {esc }}$ ). Therefore, as seen in Fig. 9, the thicker layer shows a higher effective diffusion length.

\section{CONCLUSION}

In order to interpret the recent experimental observations regarding the photophysical behavior of the metal halide perovskites, one has to consider the PR effect in the system. The photon diffusion concept we discuss in this paper shows that apparent recombination and charge transport dynamics are strongly influenced by photon absorption and re-emission. The model easily resolves the complex interplay between the photon reabsorption, carrier diffusion, and radiative $\left(\propto n^{2}\right)$ and nonradiative $\left(\propto n, n^{3}\right)$ recombination pathways. We have seen that complexity also arises from the fact that the optical design of the system (the layer thickness and interfaces) and the carrier concentration can also influence the PR impact on the optical response of the system. In agreement with the experiments, we show that the PR effect increases the apparent radiative lifetime of the carriers, and this enhancement is more pronounced for the thicker layers. While a semiconductor has an intrinsic local radiative recombination lifetime, defined by the van Roosbroeck-Shockley detailed balance relation, the photon dynamics before escape from the sample can substantially modify the experimental observations. The PL dynamics at low carrier densities is mainly governed by the carrier diffusion and the first-order nonradiative loss, and therefore, the effect of the diffusion cannot be neglected when modeling the PL decay. On the other hand, at high densities, the diffusion process becomes irrelevant, and the PR effect has a great impact on the dynamics of the decay. Interestingly, however, owing to the increase in the carrier density in the presence of the PR, the PR effect can also enhance the contribution of the nonradiative Auger recombination, and thereby, cause a decrease in the internal quantum efficiency. We also find that the PR effect can enhance the effective diffusion length of the carriers through the increase in the carrier apparent lifetime and also by transferring the carriers over long distances in the layer. In fact, we derive a general relation for the transport length that reduces to the traditional diffusion length in the case where the PR effect becomes irrelevant. We think these results can be used as a guide for further research in order to improve the optoelectronic performance of the metal halide perovskites. The results also give insights to utilize the PR effect in developing new application schemes.

\section{ACKNOWLEDGMENTS}

We acknowledge funding from MINECO of Spain under Project No. MAT2016-76892-C3-1-R and Generalitat Valenciana Project No. PROMETEOII/2014/020.

\section{APPENDIX: EFFECTIVE DIFFUSION LENGTH}

As we explain in the main text, we can write the total displacement for a carrier as

$$
\vec{L}=\vec{L}_{D}+\Lambda \vec{L}_{\mathrm{PR}}+\Lambda \vec{L}_{D}^{\prime}+\Lambda^{2} \vec{L}_{\mathrm{PR}}^{\prime}+\Lambda^{2} \vec{L}_{D}^{\prime \prime}+\cdots
$$

where $\Lambda=\eta_{\text {int }}\left(1-P_{\text {esc }}\right), L_{D} \sim \sqrt{D \tau}$ and $L_{\mathrm{PR}} \sim \alpha^{-1}$, with $\tau$ being the carrier lifetime. Therefore, the length of the vector $\vec{L}$ can be expressed as

$$
\begin{aligned}
L^{2}=\vec{L} \cdot \vec{L}= & L_{D}^{2}+\Lambda^{2} L_{\mathrm{PR}}^{2}+\Lambda^{2} L_{D}^{\prime 2}+\Lambda^{4} L_{\mathrm{PR}}^{\prime 2}+\Lambda^{4} L_{D}^{\prime \prime 2} \\
& +\cdots+\Lambda \vec{L}_{D} \cdot \vec{L}_{\mathrm{PR}}+\Lambda \vec{L}_{D} \cdot \vec{L}_{D}^{\prime}+\Lambda^{2} \vec{L}_{D} \cdot \vec{L}_{\mathrm{PR}}^{\prime} \\
& +\Lambda^{2} \vec{L}_{D} \cdot \vec{L}_{D}^{\prime \prime}+\cdots+\Lambda \vec{L}_{\mathrm{PR}} \cdot \vec{L}_{D}+\Lambda^{2} \vec{L}_{\mathrm{PR}} \cdot \vec{L}_{D}^{\prime} \\
& +\Lambda^{3} \vec{L}_{\mathrm{PR}} \cdot \vec{L}_{\mathrm{PR}}^{\prime}+\Lambda^{3} \vec{L}_{\mathrm{PR}} \cdot \vec{L}_{D}^{\prime \prime}+\cdots
\end{aligned}
$$

Now, let us consider the statistical averaging of $L^{2}$, that is, $\left\langle L^{2}\right\rangle$. We note that

$$
\left\langle L_{D}^{2}\right\rangle=\left\langle L_{D}^{\prime 2}\right\rangle=\left\langle L_{D}^{\prime \prime 2}\right\rangle=\cdots=D \tau
$$

and

$$
\left\langle L_{\mathrm{PR}}^{2}\right\rangle=\left\langle L_{\mathrm{PR}}^{\prime 2}\right\rangle=\left\langle L_{\mathrm{PR}}^{\prime \prime 2}\right\rangle=\cdots=\alpha^{-2}
$$

On the other hand, since the displacements are randomly directed, statistical averaging of other terms vanishes, that is,

$$
\left\langle\vec{L}_{D} \cdot \vec{L}_{\mathrm{PR}}\right\rangle=\left\langle\vec{L}_{D} \cdot \vec{L}_{D}^{\prime}\right\rangle=\left\langle\vec{L}_{D} \cdot \vec{L}_{\mathrm{PR}}^{\prime}\right\rangle=\cdots=0
$$

and we get

$$
\begin{aligned}
\left\langle L^{2}\right\rangle & =D \tau+\Lambda^{2} \alpha^{-2}+\Lambda^{2} D \tau+\Lambda^{4} \alpha^{-2}+\Lambda^{4} D \tau+\cdots \\
& =\left(D \tau+\Lambda^{2} \alpha^{-2}\right) \sum_{k=0}^{\infty}\left(\Lambda^{2}\right)^{k} \\
& =\frac{D \tau+\Lambda^{2} \alpha^{-2}}{1-\Lambda^{2}}
\end{aligned}
$$

Therefore, for the effective diffusion length we have

$$
L_{\mathrm{eff}} \equiv \sqrt{\left\langle L^{2}\right\rangle}=\sqrt{\frac{D \tau+\Lambda^{2} \alpha^{-2}}{1-\Lambda^{2}}}
$$

[1] M. A. Green, A. Ho-Baillie, and H. J. Snaith, The emergence of perovskite solar cells, Nat. Photonics 8, 506 (2014). 
[2] Y. Yamada, T. Yamada, L. Q. Phuong, N. Maruyama, H. Nishimura, A. Wakamiya, Y. Murata, and Y. Kanemitsu, Dynamic optical properties of $\mathrm{CH} 3 \mathrm{NH} 3 \mathrm{PbI} 3$ single crystals as revealed by one-and two-photon excited photoluminescence measurements, J. Am. Chem. Soc. 137, 10456 (2015).

[3] L. M. Pazos-Outón, M. Szumilo, R. Lamboll, J. M. Richter, M. Crespo-Quesada, M. Abdi-Jalebi, H. J. Beeson, M. Vrućinić, M. Alsari, H. J. Snaith, and B. Ehrler, Photon recycling in lead iodide perovskite solar cells, Science 351, 1430 (2016).

[4] Y. Yamada, T. Nakamura, M. Endo, A. Wakamiya, and Y. Kanemitsu, Photocarrier recombination dynamics in perovskite $\mathrm{CH} 3 \mathrm{NH} 3 \mathrm{PbI} 3$ for solar cell applications, J. Am. Chem. Soc. 136, 11610 (2014).

[5] Y. Yamada, T. Yamada, and Y. Kanemitsu, Free carrier radiative recombination and photon recycling in lead halide perovskite solar cell materials, Bull. Chem. Soc. Jpn. 90, 1129 (2017).

[6] T. Yamada, Y. Yamada, Y. Nakaike, A. Wakamiya, and Y. Kanemitsu, Photon Emission and Reabsorption Processes in $\mathrm{CH} 3 \mathrm{NH} 3 \mathrm{PbBr} 3$ Single Crystals Revealed by Time-Resolved Two-Photon-Excitation Photoluminescence Microscopy, Phys. Rev. Appl. 7, 014001 (2017).

[7] F. Staub, T. Kirchartz, K. Bittkau, and U. Rau, Manipulating the net radiative recombination rate in lead halide perovskite films by modification of light outcoupling, J. Phys. Chem. Lett. 8, 5084 (2017).

[8] T. W. Crothers, R. L. Milot, J. B. Patel, E. S. Parrott, J. Schlipf, P. Müller-Buschbaum, M. B. Johnston, and L. M. Herz, Photon reabsorption masks intrinsic bimolecular charge-carrier recombination in $\mathrm{CH}_{3} \mathrm{NH}_{3} \mathrm{PbI}_{3}$ perovskite, Nano Lett. 17, 5782 (2017).

[9] J. M. Richter, M. Abdi-Jalebi, A. Sadhanala, M. Tabachnyk, J. P. Rivett, L. M. Pazos-Outón, K. C. Gödel, M. Price, F. Deschler, and R. H. Friend, Enhancing photoluminescence yields in lead halide perovskites by photon recycling and light out-coupling, Nat. Commun. 7, 13941 (2016).

[10] R. K. Ahrenkiel, D. J. Dunlavy, B. Keyes, S. M. Vernon, T. M. Dixon, S. P. Tobin, K. L. Miller, and R. E. Hayes, Ultralong minority-carrier lifetime epitaxial GaAs by photon recycling, Appl. Phys. Lett. 55, 1088 (1989).

[11] Y. Fang, H. Wei, Q. Dong, and J. Huang, Quantification of re-absorption and re-emission processes to determine photon recycling efficiency in perovskite single crystals, Nat. Commun. 8, 14417 (2017).
[12] T. Kirchartz, F. Staub, and U. Rau, Impact of photon recycling on the open-circuit voltage of metal halide perovskite solar cells, ACS Energy Lett. 1, 731 (2016).

[13] M. A. Steiner, J. F. Geisz, I. Garcia, D. J. Friedman, A. Duda, and S. R. Kurtz, Optical enhancement of the opencircuit voltage in high quality GaAs solar cells, J. Appl. Phys. 113, 123109 (2013).

[14] S. D. Stranks, Non-radiative losses in metal halide perovskites, ACS Energy Lett. 2, 1515 (2017).

[15] J. Bisquert, The Physics of Solar Cells: Perovskites, Organics, and Photovoltaic Fundamentals (CRC Press, Boca Raton, Florida, 2017).

[16] M. Ansari-Rad and J. Bisquert, Theory of light-modulated emission spectroscopy, J. Phys. Chem. Lett. 8, 3673 (2017).

[17] S. L. Jacques and B. W. Pogue, Tutorial on diffuse light transport, J. Biomed. Opt. 13, 041302 (2008).

[18] U. Rau, U. W. Paetzold, and T. Kirchartz, Thermodynamics of light management in photovoltaic devices, Phys. Rev. B 90, 035211 (2014).

[19] See Supplemental Material at http://link.aps.org/supple mental/10.1103/PhysRevApplied.10.034062 for Escape probability calculated from two different methods, heat maps of charge density and internal efficiency, effect of the carrier diffusion and photon recycling on the carrier dynamics, and effect of the constant vs. time-dependent escape probability on the time evolution of the photon density.

[20] T. Yamada, Y. Yamada, H. Nishimura, Y. Nakaike, A. Wakamiya, Y. Murata, and Y. Kanemitsu, Fast free-carrier diffusion in $\mathrm{CH}_{3} \mathrm{NH}_{3} \mathrm{PbBr}_{3}$ single crystals revealed by time-resolved one-and two-photon excitation photoluminescence spectroscopy, Adv. Electron. Mater. 2, 1500290 (2016).

[21] Y. Yamada, M. Hoyano, R. Akashi, K. Oto, and Y. Kanemitsu, Impact of chemical doping on optical responses in bismuth-doped $\mathrm{CH}_{3} \mathrm{NH}_{3} \mathrm{PbBr}_{3}$ single crystals: Carrier lifetime and photon recycling, J. Phys. Chem. Lett. 8, 5798 (2017).

[22] T. Yamada, T. Aharen, and Y. Kanemitsu, Near-Band-Edge Optical Responses of $\mathrm{CH}_{3} \mathrm{NH}_{3} \mathrm{PbCl}_{3}$ Single Crystals: Photon Recycling of Excitonic Luminescence, Phys. Rev. Lett. 120, 057404 (2018).

[23] S. D. Stranks, G. E. Eperon, G. Grancini, C. Menelaou, M. J. Alcocer, T. Leijtens, L. M. Herz, A. Petrozza, and H. J. Snaith, Electron-hole diffusion lengths exceeding 1 micrometer in an organometal trihalide perovskite absorber, Science 342, 341 (2013). 